\title{
Dénouer les ficelles du métier
}

Pour connecter les savoirs formels et informels

Tricks of the Trade

\section{Marcelle Stroobants}

\section{(2) OpenEdition}

\section{Journals}

Édition électronique

URL : https://journals.openedition.org/tc/4625

DOI : $10.4000 /$ tc. 4625

ISSN : 1952-420X

Éditeur

Éditions de l'EHESS

\section{Édition imprimée}

Date de publication : 15 août 2009

Pagination : 164-179

ISBN : 978-2-7351-1235-7

ISSN : 0248-6016

\section{Référence électronique}

Marcelle Stroobants, « Dénouer les ficelles du métier », Techniques \& Culture [En ligne], 51 | 2009, mis en ligne le 15 juin 2011, consulté le 29 septembre 2022. URL : http://journals.openedition.org/tc/4625 ; DOI : https://doi.org/10.4000/tc.4625 


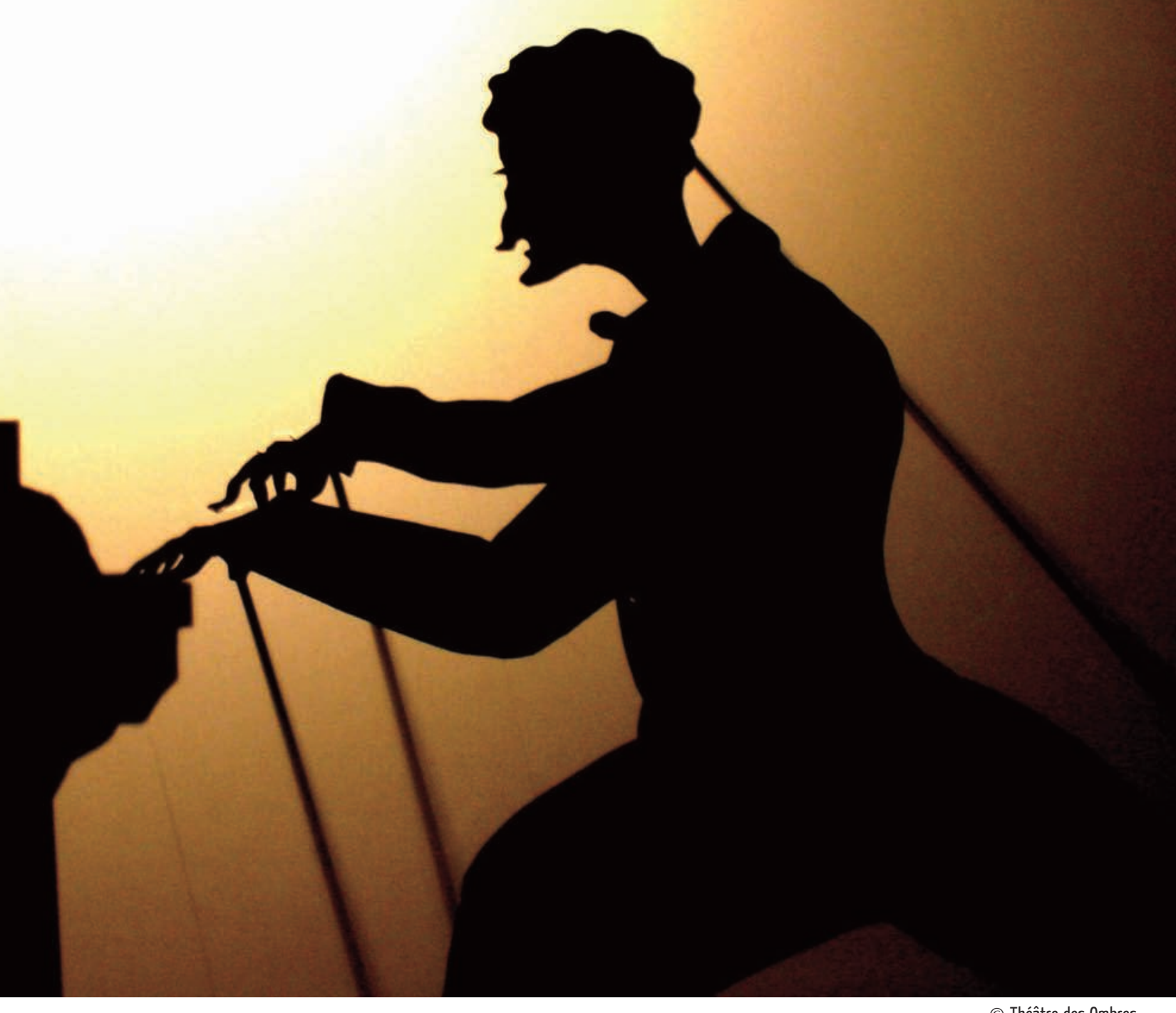

(C) Thêâtre des 0mbres 
Marcelle Stroobants

Université Libre de Bruxelles

mstrooba@ulb.ac.be
Des choses, des gestes, des mots

Techniques E Culture 51, 2008 : 164-179

\title{
DÉNOUER LES FICELLES DU MÉTIER
}

\section{pour connecter les savoirs formels et informels}

\author{
À l'école ou à l'atelier, où apprend-on les savoirs nécessaires au travail ? Et ces \\ savoirs, tellement incorporés que leur origine est oubliée, comment les mesurer \\ et avec quels effets?
}

Les connaissances impliquées dans l'action paraissent toujours en partie insaisissables, inaccessibles directement. Elles ne semblent pouvoir être évoquées que par la négative, comme second terme d'oppositions telles que savoir/savoir-faire, connaissance explicite/tacite, savoir formel/informel, théorie/pratique, etc. Ces catégories, à première vue incontournables, s'avèrent aussi encombrantes que discutables et ouvertes à la naturalisation.

Le chercheur - anthropologue ou sociologue - n'a pas à juger de la pertinence de telles catégories; pour lui, il s'agit de comprendre ce qu'elles recouvrent, comment elles ont été fabriquées, comment elles se transforment, quels en sont les enjeux et les effets. Dans les situations de compétition, sportive, artistique, scolaire ou professionnelle, dès lors qu'une évaluation est en jeu, ces oppositions deviennent stratégiques. Rarement symétriques, elles sont souvent utilisées dans la perspective de valoriser un terme par rapport à l'autre qui lui sert de repoussoir.

Dans le champ professionnel, les enjeux sont cruciaux, puisqu'il y va de la négociation de l'emploi et de la qualification à l'embauche et en cours de carrière, avec des conséquences sonnantes et trébuchantes. Dans les 
sociétés industrielles, depuis qu'existe l'obligation scolaire, les offres provenant des milieux éducatifs et les demandes des employeurs ont souvent été discordantes. D'un côté, l'école s'est donné pour mission d'ouvrir l'accès à des connaissances élargies. De l'autre, le système productif a longtemps tablé sur des spécialités directement utilisables, taillées à la mesure de fonctions pointues. Même les formations techniques et professionnelles ménagent une certaine mobilité aux futurs employés, en les préparant à un éventail d'occupations plutôt qu'à un seul poste de travail. En ce sens, la formation initiale est forcément inadéquate à l'emploi.

Or, depuis le milieu des années 1980, ces discordances se sont atténuées; des arguments venus de part et d'autre sont même entrés en résonance sur la nécessité de promouvoir des compétences peu ou pas encore formalisées sous le régime de la qualification. Du côté du système éducatif, des préoccupations utilitaristes et humanistes ont paru s'accorder dans la dénonciation commune de la « simple » transmission des connaissances. De leur côté, les employeurs, misant sur la flexibilité du marché du travail, ont renouvelé leurs exigences, faisant appel à des « ressources» humaines, plus polyvalentes, autonomes, responsables, capables d' « apprendre à apprendre » et, de surcroît, « tout au long de la vie $»^{1}$. Au-delà de ces apparentes convergences, les usages de la notion de compétence n'ont guère favorisé la reconnaissance des acquis de la formation des travailleurs; les tentatives de formalisation en vue de l'évaluation scolaire et professionnelle ont plutôt contribué à ébranler les anciens critères de qualification.

\section{Les leçons du piano}

Un énoncé aussi tranché que « la formation des compétences est bien plus que la simple transmission des connaissances » suscite d'emblée une série d'interrogations.

Pour qui et dans quelles conditions la transmission des connaissances serait-elle un acte simple? Et un acte distinct de la formation des compétences? En situation scolaire, la tâche de transmission ne brille pourtant pas par sa simplicité. N'est-on pas en train de la réduire à la communication d'informations? Et en milieu professionnel? Que savons-nous de la manière dont se forment les compétences, dont s'apprennent, se transmettent, s'acquièrent des connaissances pratiques ou des savoir-faire?

À l'écoute des travailleurs, le savoir-faire reste énigmatique, réfractaire à l'apprentissage; soit il est assimilé à un « don », soit à une habitude qui rentre à force, par compagnonnage, next to Nelly, sur le mode « tu regardes et tu fais $\%$.

Les très riches témoignages recueillis par Studs Terkel, au début des années 1970, abondent dans ce sens. Par exemple, Babe Sec Oli, caissière dans un supermarché depuis près de trente ans, convertit immédiatement ses acquis en une aptitude: 


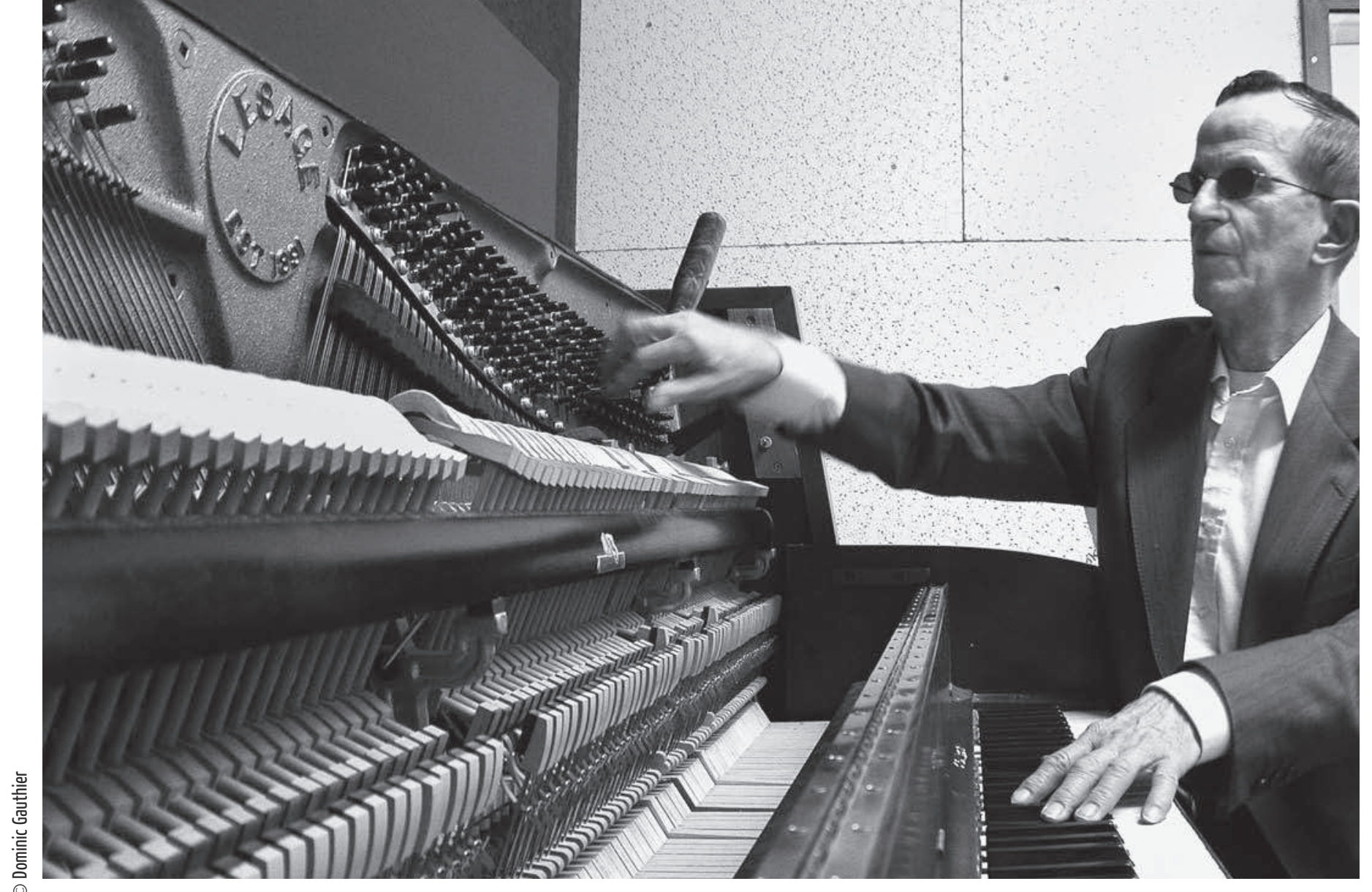

« Vous apprenez les prix par cœur en quelque sorte. C'est un don » (Terkel 2005 : 238).

Plus nettement encore, Eugene Russell, depuis quinze ans accordeur de piano «par vocation », avance:

« Je ne pense pas qu'on puisse apprendre à accorder un piano. Je dirais que c'est surtout de la pratique. Vous vous jetez à l'eau et ensuite, c'est la pratique, jusqu'à ce que ça devienne une chose naturelle pour vous » (Terkel 2005 : 276).

Demandons à n'importe quel automobiliste chevronné si l'accélérateur se trouve à gauche ou à droite du frein, il y a de fortes chances qu'il hésite à répondre à cette question alors que son corps n'hésite généralement pas à réagir, dans le feu de l'action. Loin de mettre en doute sa maîtrise de la conduite automobile, son hésitation suggère qu'il connaît « trop » bien sa machine, qu'il fait corps avec elle, que les pédales sont les prolongements de ses propres pieds.

Dès 1877, Samuel Butler développait, dans La Vie et l'habitude, ce paradoxe selon lequel le comble du savoir se pratique en ignorance de cause tandis que le novice n'ignore rien de ses propres tâtonnements. Le pianiste, précisément, semble avoir beaucoup de mal à reconstituer les embûches d'un morceau qu'il maîtrise parfaitement: « on dirait qu'il sait ce morceau trop bien pour savoir qu'il le sait, et que les seuls passages qu'il a conscience de savoir sont ceux qu'il ne sait pas aussi bien » (Butler 1922 : 19). Avant d'avoir appris, on ne 
voit que les obstacles à surmonter; par la suite, l'habitude masque les ressorts de l'habileté, le savoir-faire devient imperceptible, comme englouti.

Un siècle plus tard, Michel Verret, sociologue de la classe ouvrière, envisage l'existence de savoirs "non scolarisables ", tels que les savoirs initiatiques, les savoirs « aristocratiques », les savoirs « personnels » et « empiriques » avant de reprendre cette même idée: « un savoir appris est un savoir qu'on ne sait plus qu'on sait, qu'on a sans le savoir » (Verret 1975: 140 sqq).

Dès lors, savoirs formels et informels n'apparaissent plus comme des catégories stables et distinctes, mais comme des étapes ou des modes de connaissance différenciés, se dérobant ou non à la scolarisation, plus ou moins perceptibles.

Les ficelles du métier, réputé intellectuel ou manuel, les tours de main et les coups d'oil experts, relèvent donc d'un registre ineffable, de ce que le philosophe Michael Polanyi (1958) a appelé la dimension tacite de la connaissance. Dans la mesure où ces savoirs tacites sont indicibles et échappent même aux principaux intéressés, on pourrait penser qu'ils ne concernent pas la sociologie ou pas encore. On pourrait même les renvoyer dans ce que Bruno Latour a appelé le " plasma », cette zone interstitielle, encore inconnue où baigne de l'invisible en attente d'explicitation, « ce qui n'est pas encore formaté, pas encore mesuré, pas encore socialisé » (Latour 2006 : 351).

Où situer, par exemple, ces savoirs aristocratiques envisagés par Verret? La réponse est à nouveau anticipée par Butler, à propos de « ces jeunes gens de l'aristocratie et de la haute bourgeoisie anglaises qui vivent beaucoup au grand air ». Leur «science » infuse, leur aisance, et leurs dons apparents ne se transmettent pas par l'instruction - « ils ne lisent jamais »- mais par une dot, un appareil coûteux, une « expérience pratique héréditaire remontant à plusieurs générations » (Butler 1922 : 46). Cette expérience évoque irrésistiblement le concept d'habitus, proposé par Bourdieu, et dont Latour reconnaît l'excellence, à condition, dit-il, de dégager ce concept de son corpus théorique (Latour 2006 : 307 n. 31).

À partir du paradoxe du pianiste, il faudrait alors envisager des situations instables, comportant des mouvements d'intériorisation, un devenir tacite, autant que des mouvements d'extériorisation du plasma. Tout adulte dont la jambe a été fracturée et immobilisée dans le plâtre pendant plusieurs semaines, peut faire cette expérience étrange d'avoir à réapprendre à marcher. Car cette rééducation n'attend pas seulement une revalidation musculaire, mais demande à s'interroger sur la manière de poser le pied, de le dérouler, tout en rendant hommage à ces palpeurs méconnus, les orteils. Il s'agit de réinventer la poudre de ce savoir-faire, ou, avec l'aide d'un instructeur, de sortir ce savoir-faire du plasma où il retournera tôt ou tard.

Pour Bruno Latour, le plasma informel fournirait « les ressources nécessaires au déroulement de chaque action » (Latour 2006 : 352), ressources de toutes provenances, acquises une par une, constituant autant de bouts de savoir-faire. À la lumière d'une métaphore internautique, il dénomme ces bribes de compétences des " plug-ins », à l'instar des logiciels téléchargeables permettant d'augmenter les fonctionnalités d'un programme 
particulier. Il y a une multitude de « clichés culturels » à absorber pour devenir capable d'interpréter et d'exprimer une opinion, d'entretenir une conversation, d'aventurer une déclaration d'amour, de se débrouiller dans un supermarché, d'adopter une démarche, une voix, des vêtements de circonstances, etc. Chacune des microcompétences qui composent notre singularité aurait ainsi été puisée dans son site formaté. Saluant la voie de l'extériorisation ouverte par William James, Latour nous invite ainsi à comprendre l'acquisition de notre intériorité comme rassemblement de multiples attachements avec l'extérieur (Latour 2006 : 314-316).

Pour pouvoir se prévaloir de ces multiples connexions ad hoc, habitudes incorporées, habiletés incarnées, encore faut-il qu'elles soient habilitées, estampillées par la voie de l'évaluation sociale.

\section{Une technique peut en cacher une autre}

L'étude des savoir-faire n'occupe qu'une place restreinte et récente en sociologie du travail. Cette spécialité, sensible aux structures de domination et d'exploitation n'a longtemps abordé les connaissances professionnelles que par défaut ou par contraste. Sous l'angle du salariat et de la division du travail, l'apprentissage - devenir forgeron en forgeant - semble révolu en même temps que les structures des corporations. Le taylorisme a souvent été envisagé comme une machine de guerre dirigée contre les travailleurs et destinée à les déposséder de leur savoir-faire. Dans leur cadre originel américain, au tournant du XX ${ }^{\mathrm{e}}$ siècle, les formalisations tayloriennes ont effectivement pu porter atteinte aux monopoles des ouvriers professionnels et des gens de métier. En même temps, pour les vagues de migrants de souche rurale et dénués d'expérience de l'usine, les procédures rationalisantes de Taylor pouvaient servir de recettes. Sous cet angle-là, le taylorisme apparaît comme un mode de vulgarisation des connaissances industrielles, une traduction certes déformante et destinée à intensifier le travail, mais susceptible en même temps d'ouvrir le marché du travail à une maind'œuvre qui n'y avait pas accès.

De son côté, l'ergonomie, assumant l'héritage de l'analyse du travail, a pu appréhender l'habileté, ou le savoir-faire, comme intériorisation de la technique et, à la suite de Leroi-Gourhan, réserver la dénomination de technique à un procédé pouvant être transmis. En ce sens, la technique serait intrinsèquement sociale et le savoir-faire intrinsèquement non transmissible (Leplat \& Pailhous 1981 : 276).

Pourtant, dès le milieu des années 1980, le savoir-faire réapparaît sur le terrain industriel et sous la plume de certains chercheurs. On peut distinguer au moins deux courants d'études qui annoncent, chacun à leur façon, l'importance des compétences.

Un premier courant vient balayer - comme un vent de fraîcheur dans le paysage du travail - les travaux antérieurs qui avaient pu dénoncer la 
dégradation du travail et des qualifications. Les ouvriers ne sont plus dépeints en robots asservis, mais redeviennent habiles, savants, bricoleurs, créateurs. Toute une série d'observations fines, souvent assistées par des ergonomes, attestent alors des stratégies insoupçonnées, des formes d'inventivité et d'initiative, préalablement négligées. Dans cette perspective, les salariés s'avèrent plus compétents qu'on ne le croyait. Cette réfutation s'adresse rétrospectivement aux autres chercheurs qui avaient pu sous-estimer la richesse des interventions informelles, mais aussi aux rationalisateurs et aux concepteurs de machines. De là à redécouvrir que les qualifications « conventionnelles » ne rendent pas justice à tous ces savoir-faire et à juger plus « vraies » toutes ces compétences ineffables, il n'y a qu'un pas, que certains auteurs franchissent allègrement. D'autres, en revanche, hésitent à endosser une posture d'arbitrage qui risquerait, en outre, de naturaliser les conventions de qualification.

Le deuxième courant de recherche qui se développe dans la foulée tend plutôt à soutenir que des compétences différentes et plus importantes qu'avant seraient mobilisées au travail. Ici, l'accent est surtout mis sur des capacités de communication, des qualités de gestion, des savoir-être, voire des « compétences sociales ». Ce courant-ci se place dans une perspective normative, au service d'une stratégie de gestion individualisée du personnel.

Il n'existe manifestement aucune unanimité sur la définition de ces compétences qui seraient plus importantes que prévu ou plus importantes qu'avant. La seule constante observable relève de la catégorisation plutôt que du contenu. À la fin de cette décennie, un certain consensus semble établi sur le fait que les compétences désignent la capacité à mobiliser des « savoirs, des savoir-faire, des savoir-être ». Ce fameux trio, qu'on retrouve aussi dans la sphère éducative, s'est lui-même élaboré à coup de (re)découvertes progressives. "Non seulement» le travail mobilise des savoirs, « mais aussi » des savoir-faire et « non seulement » des savoir-faire, «mais encore » des savoirs non techniques, sociaux ou des savoir-être. Chacun de ces « non seulement » fonctionne un peu comme un repoussoir de l'évidence. " Non seulement des savoirs » laisse entendre qu'il y a plus dans la pratique professionnelle que ces évidentes connaissances formelles, théoriques, transmises par la formation initiale. « Non seulement des savoirfaire » suggère qu'au-delà des procédures et des opérations techniques, il y aurait une sorte de supplément d'âme, des manières d'être, du social, insaisissable, plus informel que tout autre. On conviendra que cette trilogie peut s'appliquer à tout, y compris au passé. On continuera à se demander ce que pourrait être une compétence qui ne soit pas sociale.

La question qui importe ici serait plutôt de comprendre la soudaineté de cette découverte. Comment tous ces savoir-faire tacites ont-ils pu brusquement sortir du plasma?

On se souvient que la décennie 1980 a été marquée par les applications de la microélectronique et que de nombreux programmes de recherche se sont orientés dans ce sens. La sociologie du travail s'est trouvée une fois de plus confrontée aux déterminations techniques. Or, si les TIC, les technologies de l'information et de la communication, ont pu jouer un 
rôle dans le succès de la notion de compétence, c'est bien celui de révélateur. Car toutes ces sortes de savoir-faire, de savoirs tacites, de talents insoupçonnés se manifestent d'abord par contraste avec les tentatives de les simuler artificiellement. Les ingénieurs tayloriens avaient entrepris de rationaliser les méthodes pour accélérer le rythme de travail. Les ingénieurs de la connaissance tentent à leur tour de formaliser tout ce qui ne l'est pas encore pour élaborer des systèmes experts, des automates programmables et des robots. Des gestes apparemment simples, des tours de mains, des coups d'œil, autant d'actes d'apparente routine se sont alors avérés plus complexes que prévu, peu susceptibles de se prêter à une décomposition en procédures programmables.

Du coup, le blason des exécutants a été redoré. L'intelligence des travailleurs est redevenue d'autant plus visible à mesure que les ambitions de l'intelligence artificielle se réduisaient ou empruntaient d'autres voies.

Dans le second courant de recherche, moins exotique, les compétences se déclinent aussi en relation avec les machines. Non par concurrence, mais par complémentarité. Pour piloter ou surveiller la production assistée par ordinateur, les qualités attendues des opérateurs se voient modernisées, déclinées dans le vocabulaire des technologies informatiques: savoir communiquer, savoir décoder et transmettre de l'information, en termes d'heuristique et de résolution de problème. C'est aussi l'époque où les sciences cognitives se diffusent dans les milieux professionnels et de la formation, notamment par le truchement des ergonomes, des consultants et des technologues.

En définitive, la manière de percevoir les technologies, les revirements et les surprises qu'elles ménagent ont bien servi de révélateur. Des habiletés invisibles transparaissent, résistent, sont amplifiées avant tout parce qu'on a changé de manière de voir. Ces qualités-là ne sont ni plus ni moins « réelles » ni plus ni moins « représentées » que celles qui avaient été entrevues sous l'angle des qualifications. Toutefois, les qualités qui font la différence entre machines et humains risquent de ne pas être aisément valorisables sur le marché du travail dès lors qu'elles sont communément partagées, banales chez les humains.

En tout état de cause, le second courant de recherches l'a emporté, inaugurant une ère de gestion plus flexible, d'évaluation individualisée et de formalisation des compétences.

\section{La question fait le larron}

Bon nombre de talents, de tours de main ou de ficelles demeurent engloutis, implicites, incorporés à une pratique personnelle ou à un acte singulier. Les processus d'acquisition ou d'apprentissage restent peu explorés, les acquis de l'expérience paraissent surgir de nulle part. Les savoir-faire semblent alors « naître du procès de travail », sans rapport avec la formation 
antérieure, alors même que les salariés sont plus instruits que jamais. Dans une perspective de mobilité accrue, on s'attend à ce qu'ils puissent s'abstraire de la situation qui les aurait fait naître, tablant sur d'hypothétiques compétences « transférables».

Certains chercheurs, misant sur l'expérience acquise sur le tas, ont avancé l'hypothèse que celle-ci pouvait générer de précieuses connaissances techniques, sans commune mesure avec la formation initiale, sans rapport avec l'instruction scolaire (Bernoux et al. 1983). Pour rendre la parole aux principaux intéressés, ils ont décidé de renoncer aux entretiens formels, de remplacer le système de questions-réponses par un mode de communication moins verbal. Ces enquêteurs ont donc demandé à une cinquantaine d'ouvriers de dessiner « leur » machine et de commenter son fonctionnement. Le matériel récolté s'est avéré très riche mais difficile à interpréter sans le concours d'un professeur de dessin technique. Finalement, les résultats ont infirmé l'hypothèse de travail, la connaissance de l'usage des machines semble tout de même aller de pair avec la formation technique initiale. La recherche s'arrête là, alors que les résultats laissent place à d'autres interprétations non moins intéressantes.

À première vue, tout semble se passer comme si les savoirs formels et informels étaient directement et non inversement proportionnels. Les connaissances scolaires de base habiliteraient à tirer parti de la pratique. Dans ce cas, le savoir formel ne serait plus opposé au non formel, mais y donnerait accès.

Cependant, le dessin n'échappe pas non plus à la logique scolaire, il a pu privilégier l'instruction au détriment de l'expérience. La pratique des machines a pu rester inaccessible par la voie des techniques graphiques. Les savoir-faire ont pu résister au dessin comme ils pouvaient se montrer récalcitrants à l'interrogation verbale. Comment trancher?

Dans l'entreprise, la question ne se pose pas. Soit les travailleurs sont jugés au pied du mur, d'après leurs performances, soit ils sont sélectionnés sur d'autres critères formels; dans les deux cas, les savoir-faire informels restent engloutis dans le plasma, comme s'ils n'avaient aucune existence.

Cette dernière éventualité s'est trouvée confirmée sur mon propre terrain, lors d'une rencontre avec un responsable de la formation d'une entreprise de fabrications métalliques qui déplorait l'ignorance d'une majorité de travailleurs pourtant habitués de longue date à manier des machines-outils.

- Vous connaissez la différence entre le tour et la fraiseuse: dans le premier cas, c'est la pièce qui tourne, et dans le second, c'est l'outil qui tourne. Or, parmi les ouvriers qui travaillaient depuis longtemps sur ces machines, beaucoup ne le savaient pas.

— Comment vous en êtes-vous aperçu?

— On leur a demandé de répondre à un questionnaire (Stroobants 1993 : 216). 
Comment des opérateurs capables de piloter des tours et des fraiseuses, de fixer les outils, de charger les pièces, de régler les vitesses et les avances des uns ou des autres etc., comment pourraient-ils ne pas discerner ce qui tourne sous leurs yeux? Que s'est-il donc passé? À nouveau, la technique d'évaluation - et tout particulièrement cette question - a pu filtrer les réponses en fonction de la scolarité. Une minorité ayant tâté du métier d'élève assez longtemps et assez récemment a pu deviner les attentes du concepteur du questionnaire. Pour tous les autres, la réponse ne s'imposait pas parce que cette différence-là n'était pas ou plus pertinente pour eux. « La » différence entre « le » tour et « la» fraiseuse ne crevait pas les yeux, ou n'avait pas plus de sens que toutes les autres distinctions entre les machines-outils de diverses générations, de différentes marques, de différents types qu'ils côtoyaient tous les jours. La « bonne » réponse était une réponse de principe, une réponse scolaire, une réponse sans rapport avec la pratique. Autrement dit, la compétence évaluée est la compétence à réussir telle épreuve, en l'occurrence, à fournir telle réponse à telle question, sans impliquer quelque autre habileté.

La petite et la grosse fraiseuses (Bernoux et al., 1984)

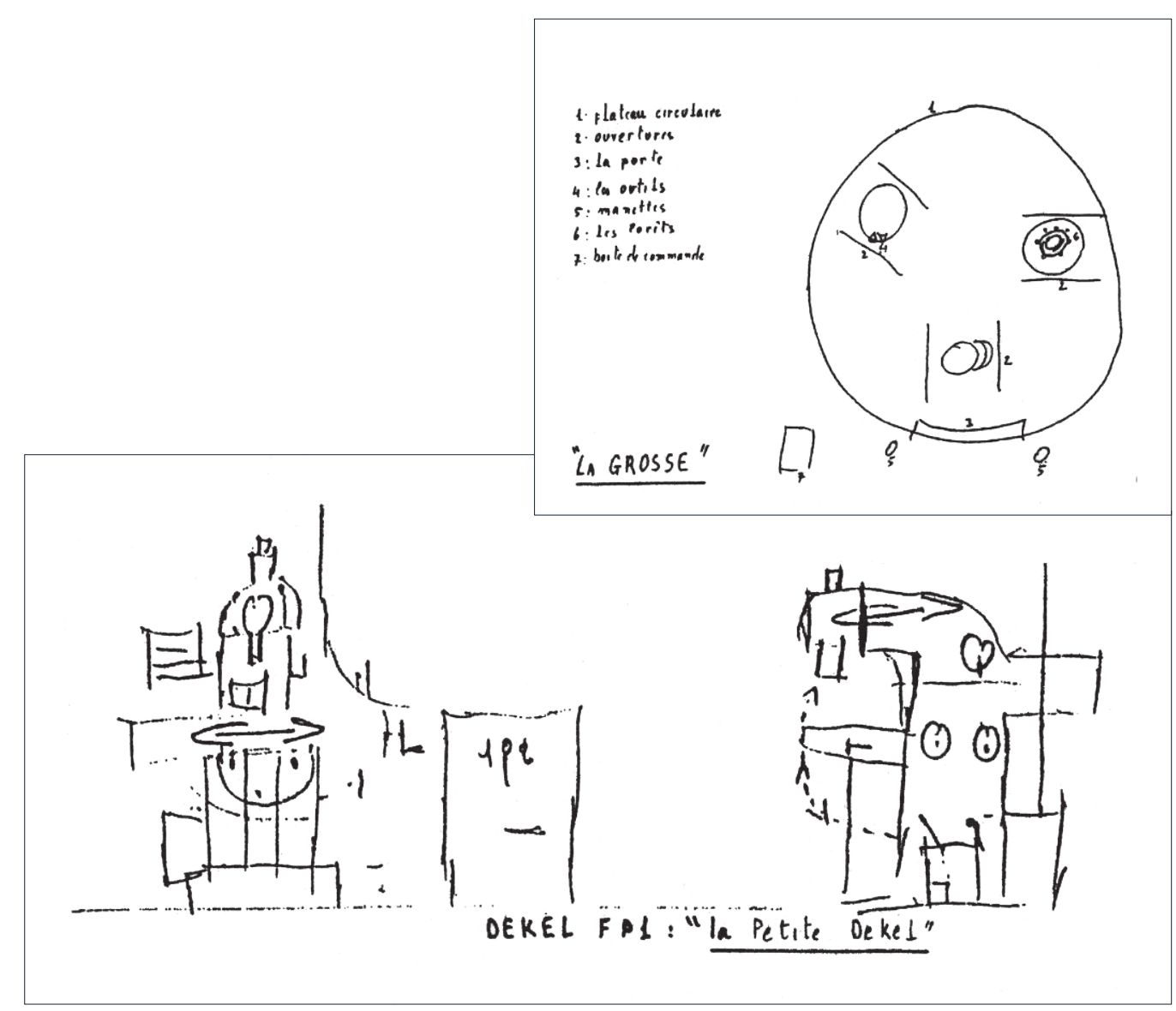




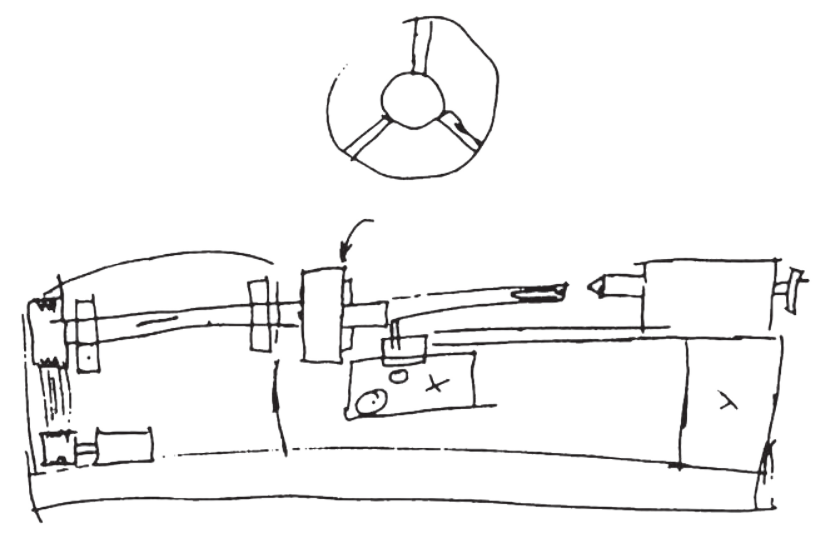

Tour semi-automat ique classique
Vue de l'intérieur

(Fig. 3)

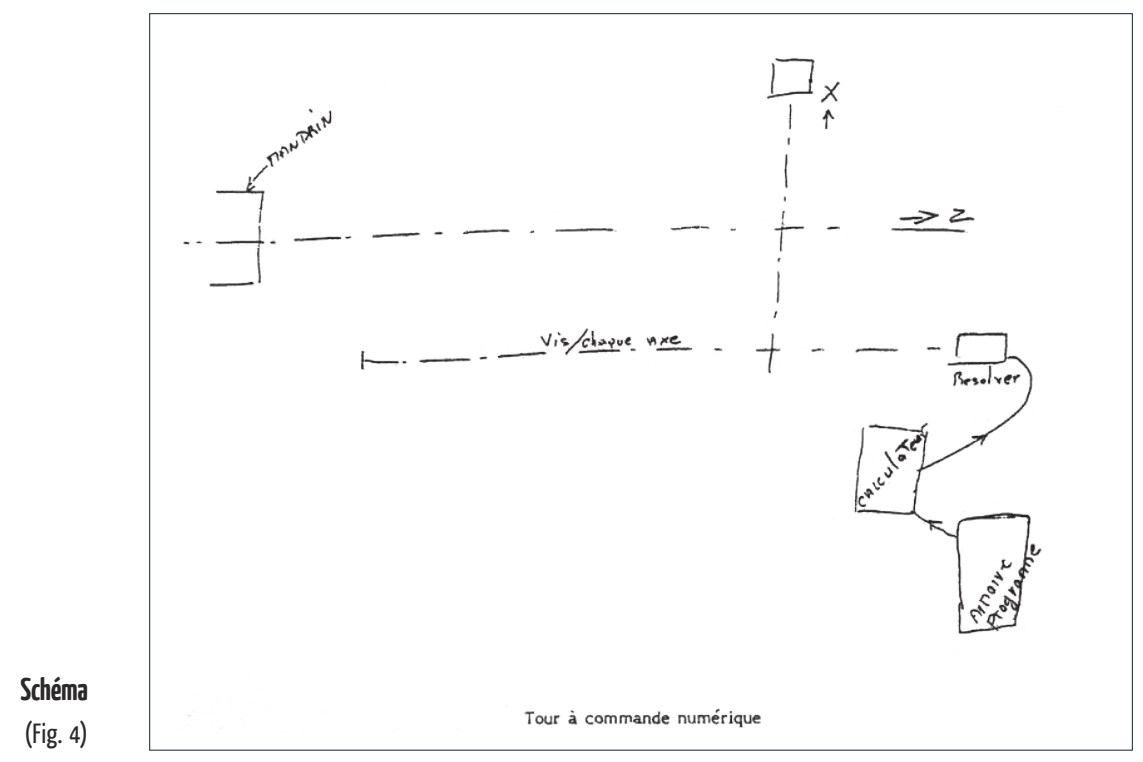

Vue d'en haut

(Fig. 5)

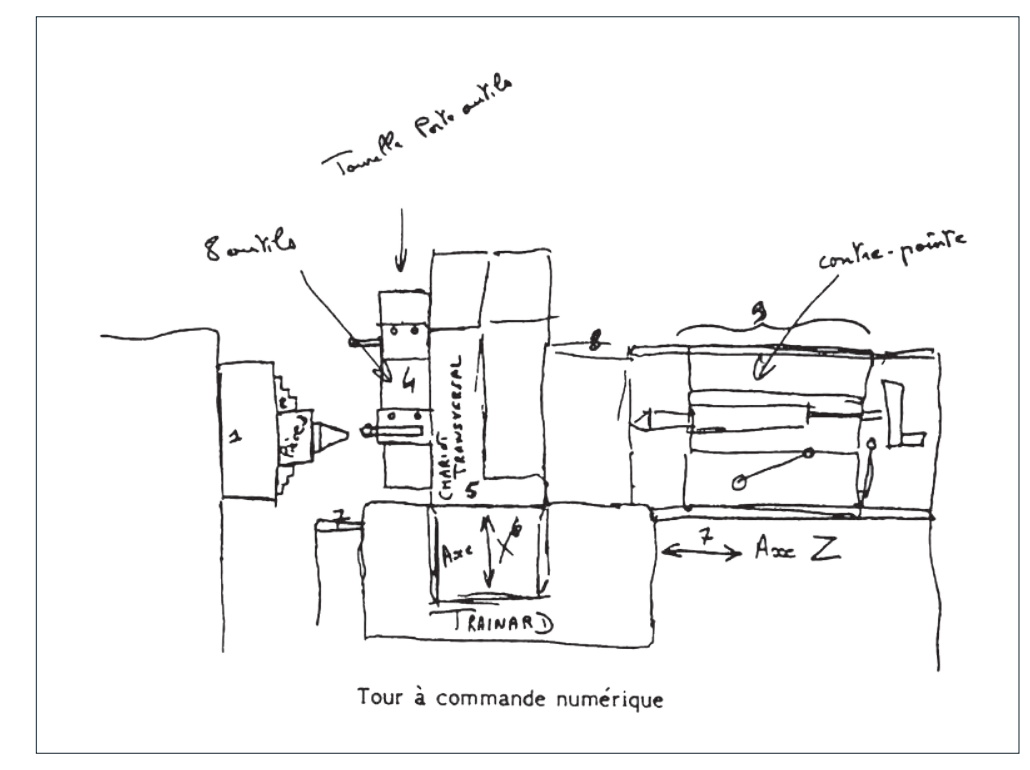




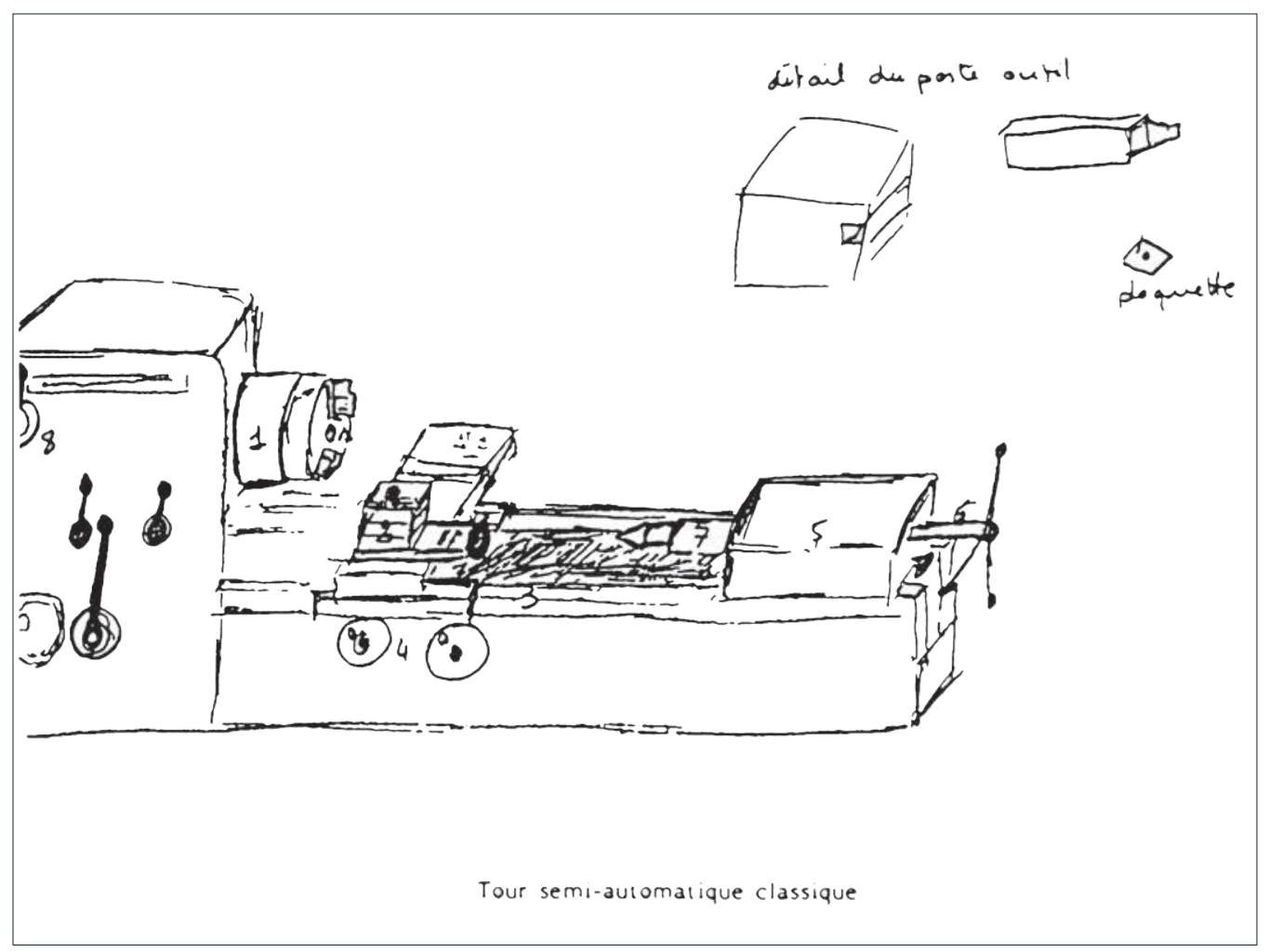

Au début des années 1980, époque où les entreprises parlaient beaucoup de gisements de ressources humaines, des sociologues du travail ont demandé à une cinquantaine d'ouvriers

de dessiner « leur » machine pour en expliquer le mode d'emploi (Bernoux et al., 1984). Deux tiers des croquis réalisés se distribuaient en quatre types, allant du plus abstrait (fig. 4) au plus réaliste (fig. 6) en passant par des points de vue plus ou moins distants (fig. 3 et 5). Les chercheurs ne s'attendaient pas à ce que le dessin, comme l'écrit, aille de pair avec une formation scolaire en mécanique.

(Fig. 2, 3, 4, 5 et 6) 
Sur le marché du travail, dans toute compétition, une compétence invisible, incommunicable, ineffable devient assimilable à une incompétence pure et simple. Un savoir-faire, une compétence n'ont d'existence sociale que s'ils sont évalués comme tels, que ce soit « au pied du mur», comme le bon maçon ou que ce soit par des procédures formelles.

Faute d'avoir pu trouver, au début des années 1990, des compétences directement identifiables, sans les repères de l'ancienneté ou de la scolarité, il a bien fallu les « inventer », c'est-à-dire les présumer à partir de systèmes d'évaluations de performances et de comportements, élaborés par des consultants et donc peu contrôlables.

\section{Rebondissement}

À première vue, les deux exemples - celui du tour et de la fraiseuse et celui du dessin de la machine - paraissent confirmer que les savoir-faire impliqués méritent bien d'être qualifiés d'informels puisqu'ils ne se laissent pas capturer par le formalisme des interrogations scolaires. La difficulté semble se réduire à un pur problème méthodologique. En conséquence, il suffirait de les interroger autrement, dans l'action, par exemple, et sous le regard des pairs. Cette perspective tendrait à corroborer l'opposition initiale entre des catégories de connaissances accessibles par le formalisme moderne et d'autres qui, à la manière artisanale, ne se laissent éprouver que dans la performance. De la sorte, la stratégie du recruteur, assimilant l'indicible à l'inexistant, se trouverait aussi confortée. Et il ne resterait plus qu'à étendre aux compétences, la fameuse formule du testeur: la compétence, c'est ce que la société évalue!

Il reste, cependant, ce détail prometteur, susceptible d'entrouvrir la boîte noire de l'apprentissage. Car dans le cas du dessin de la machine, la formation technique initiale semble compatible avec la pratique et même en favoriser l'accès. Sous cet angle, l'expérience incorporée n'apparaît plus forcément comme une qualité distincte, mais comme une dimension d'une dynamique, une «dimension tacite » de la connaissance, pour reprendre la formule de Polanyi.

Si cette dimension tacite est inhérente à la connaissance en action, elle devrait aussi être évoquée dans d'autres performances, y compris dans la mise en œuvre de savoirs réputés aussi abstraits que ceux des sciences expérimentales. En effet, considéré de l'intérieur, l'exercice des sciences naturelles ou exactes s'avère aussi requérir un art implicite et des savoirfaire très concrets. On estime même qu'il faut une dizaine d'années de pratique scientifique pour transformer un chercheur fraîchement diplômé en expert capable de comprendre et maîtriser son domaine de spécialité (Stengers \& Bensaude-Vincent 2003 : 360-362).

Les meilleurs manuels ne parviennent pas à mettre en scène le corps à corps qu'implique l'acquisition des ficelles d'un métier, y compris celles 
du chercheur. Comme la caissière Babe, le chimiste confirmé pourrait invoquer un don pour désigner cette seconde nature - bel et bien acquise - qu'il incarne. Un mathématicien, un professeur de dissertation, un promoteur de thèse seront prêts à partager l'avis de l'accordeur Eugene: il y a des pratiques qui ne s'apprennent pas. Ce que les uns et les autres laissent ainsi entendre, c'est que cela ne s'apprend pas comme ça, c'està-dire, pas comme on suivrait un mode d'emploi, une méthode ou une instruction. Mais cela s'apprend autrement, « à force » et par immersion; il s'agit, dit l'accordeur, de « se jeter à l'eau » et même de «faire corps » avec le milieu. Et ces forces en action sont si activement transformatrices qu'à l'issue de cette dynamique rien ne sera plus comme avant. L'apprenti devenu habile a si bien métabolisé les phases de sa transformation qu'il n'en a pas forcément gardé le souvenir. Posée en cours d'action, la question de la marche à suivre pourrait bien être plus perturbante qu'habilitante. Le problème n'est plus celui de la méthode adéquate mais devient ici un problème fabriqué par la méthode.

Si l'apprentissage et son corollaire, la transmission des connaissances, ne constituent pas des actes simples, c'est au moins parce que ces processus demandent à associer ce que la normalisation des formations tend explicitement à disjoindre, matière et manières.

Dans cette perspective, les tentatives de former des compétences génériques et transférables, abstraction faite du milieu qu'elles associent, loin de contribuer à « apprendre à apprendre » risquent plutôt de compromettre tout apprentissage.

\section{NOTES}

1. Voir par exemple le rapport de la Table Ronde des Industriels Européens (ERT), publié en février 1995 : http://www.ert.be/working_group.aspx?wg=15. La même année, le livre blanc de la Commission européenne, enseigner et apprendre, vers la société cognitive, reproduit ces injonctions, citant à l'appui des extraits de ce rapport. Voir http://ec.europa.eu/ education/doc/official/keydoc/lb-fr.pdf.

2. C'est à Alfred Binet, inventeur malgré lui du Q.I. au début du XX $X^{\mathrm{e}}$ siècle, qu'on attribue cette boutade : « l'intelligence c'est ce que mesure mon test». 


\section{RÉFÉRENCES}

Bernoux Ph., Magaud J., Raveyre M.-F., Ruffier J., Saglio J. \& Villegas G., 1983, « Les connaissances des salariés sur les machines qu'ils utilisent ou pourraient utiliser », Recherches économiques et sociales 8 : 95-114.

Bernoux Ph., Magaud J., Raveyre M.-F., Ruffier J., Saglio J. \& Villegas G., 1984, « Qui connaît les machines? Les connaissances des salariés sur les machines qu'ils utilisent ou pourraient utiliser », rapport de recherche du Groupe Lyonnais de Sociologie Industrielle, Commissariat général du Plan.

Butler S., 1922 [1877], La Vie et l'habitude. Paris, Nouvelle Revue Française.

Latour B., 2006, Changer de société. Refaire de la sociologie. Paris, La Découverte.

Leplat J. \& Pailhous J., 1981, «L'acquisition des habiletés mentales: la place des techniques », Le Travail humain $2: 275-282$.

Polanyi M., 1958, Personal Knowledge. Towards a Post-Critical Philosophy. London, Routledge and Kegan Paul.

Stengers I. \& Bensaude-Vincent B., 2003, 100 mots pour commencer à penser les sciences. Paris, Les empêcheurs de penser en rond.

Stroobants M., 1993, Savoir-faire et compétences au travail au travail. Une sociologie de la fabrication des aptitudes. Bruxelles, Éditions de l'Université de Bruxelles.

Terkel S., 2005 [1972], Working. Histoires orales du travail aux États-Unis. Paris, Éditions Amsterdam.

Verret M., 1975, Le Temps des études. Paris, Honoré Champion. 


\section{RÉSUMÉ}

Dénouer les ficelles du métier. La qualification professionnelle et les critères qui lui sont associés ont été fortement dépréciés, depuis une vingtaine d'années, sur le marché de l'emploi et dans les milieux de la formation, au nom de compétences « informelles », mobilisées au travail. Celles-ci sont vantées par opposition à la « simple » transmission des connaissances. Sur le terrain, les gens de métiers continuent à affirmer que le savoir-faire ne s'apprend pas ou pas « comme ça », qu'il s'apparente à un don se développant par la pratique. Des stratégies managériales, des préoccupations pédagogiques, des enquêtes sociologiques, des témoignages des principaux intéressés concourent donc à renforcer l'opposition entre savoirs formels et informels. Encore faut-il envisager le processus qui aboutit à distinguer ces deux catégories de savoirs. Car si le savoir-faire résiste à la formalisation, le savoir formel ne résiste pas forcément au savoir-faire. Certaines recherches suggèrent ainsi que la formation scolaire peut ouvrir l'accès à la pratique. Quant aux connaissances académiques, elles ne se mettent pas en œuvre non plus sans cultiver un art implicite. Ni l'inculcation ni l'application d'un mode d'emploi ni même la simple immersion ne suffisent à générer du savoir-faire. Au-delà de l'alternative entre un don ou bien une transmission par instruction, l'apprentissage semble demander une intense activité de mise en compatibilité entre des ingrédients disparates. Le milieu social, l'environnement professionnel, la situation de travail sont riches de ressources techniques susceptibles d'être convoquées dans ce processus. Malgré l'intensité de cette activité, un tel apprentissage ne laisse pas plus de souvenir que des premiers pas ou des premiers mots, lorsque cette transformation va sans dire. En l'absence d'enjeu social explicite, la connaissance semble s'évanouir comme un savoir nul et non avenu. En revanche, les épreuves sociales, les évaluations, les qualifications constituent autant de repères susceptibles de garder la mémoire de tels épisodes et d'intervenir dans leur déroulement. Ainsi construite, la distinction formel/informel pourrait - si elle est présentée comme un ingrédient naturel - perturber la formation de compétences.

\section{ABSTRACT}

Tricks of the Trade. For approximately twenty years, qualification criteria have been strongly underestimated, on the labour market and in training, in the name of « informal » competencies, required on the job. These are praised in contrast with « simple » knowledge transmission. On the field, professionnals themselves keep claiming that skills are growing by practice, like a gift not really learned. Managerial strategies, sociological studies, educational concerns and direct witnesses are thus all strengthening the opposition between formal and informal knowledges. Yet, one should consider the process leading to this distinction. If know-how resists to formal expression, the reverse is not necessarily true. Some researches show, for instance, that education opens access to practice; even academic knowledge, put into practice, is nurturing an implicit art. Neither guidelines nor a simple immersion are sufficient for generating know-how. Beyond the alternative between gift and instructions, learning process is requiring an intense activity to create compatibility between heterogeneous ingredients. Social background, professional environment, working situation are rich in technical resources available for this process. In spite of its intensity, this type of learning does not seem to be remembered anymore than one's first steps or first words, when it is implicit. Whitout any direct social issue, knowledge seems to vanish. On the other hand, social tests, assesments, qualifications are landmarks susceptible to influence learning processes and to preserve the memory of such episodes. The formal/informal distinction, being constructed, could become rather disturbing when presented as a natural input of training.

\section{MOTS CLÉS}

Apprentissage, compétence, connaissance tacite, évaluation, formel, informel, savoir-faire, qualification.

\section{KEYWORDS}

Learning, competency, tacit skill, assessment, format, informal, know-how, qualification. 